Pres. 2009 Nuclear Science Symp., Orlando, FL

25-31 October (2009)

\title{
A SEALED, UHV COMPATIBLE, SOFT X-RAY DETECTOR UTILIZING GAS ELECTRON MULTIPLIERS ${ }^{*}$
}

\author{
Neil A. Schaknowski and Graham C. Smith \\ Brookhaven National Laboratory \\ Upton, NY 11973-5000
}

December, 2009

\begin{abstract}
${ }^{*}$ This manuscript has been authored by Brookhaven Science Associates, LLC under Contract No. DC-AC02-98CH10886 with the U.S. Department of Energy. The United States Government retains, and the publisher, by accepting the article for publication, acknowledges, a world-wide license to publish or reproduce the published form of this manuscript, or allow others to do so, for the United States Government purposes.
\end{abstract}




\section{DISCLAIMER}

This work was prepared as an account of work sponsored by an agency of the United States Government. Neither the United States Government nor any agency thereof, nor any of their employees, nor any of their contractors, subcontractors, or their employees, makes any warranty, express or implied, or assumes any legal liability or responsibility for the accuracy, completeness, or any third party's use or the results of such use of any information, apparatus, product, or process disclosed, or represents that its use would not infringe privately owned rights. Reference herein to any specific commercial product, process, or service by trade name, trademark, manufacturer, or otherwise, does not necessarily constitute or imply its endorsement, recommendation, or favoring by the United States Government or any agency thereof or its contractors or subcontractors. The views and opinions of authors expressed herein do not necessarily state or reflect those of the United States Government or any agency thereof. 


\title{
A Sealed, UHV Compatible, Soft X-ray Detector Utilizing Gas Electron Multipliers
}

\author{
Neil A. Schaknowski, Graham C. Smith, Senior Member, IEEE, B. Yu and Daniel A. Fischer
}

\begin{abstract}
Abstrac-An advanced sof $X-1$-ry detector has been designed and fabricated for use in synchrotron experiments that urilize Xray absorption spectroscopy in the study a widd range af materials properties. Fluorescence $\mathrm{X}$-rays, in particular $\mathrm{C}_{\mathrm{K}}$ at $277 \mathrm{eV}$, are converted in a low pressure gas medium, and charge multiplication occurs in two gas electron multipliers, fabricated inwounse from glass reinetorced laminate, to enable single photon counting. The detector satisfies a number of demanding characteristics often required in synehrotron environments, such as UHV compatibility, compactuess, long-term stability, and energy resolving capabillity.
\end{abstract}

\section{INTRODUCTION}

GOFT X-rays are an important probe in studies of a wide range of materials, and measurement of $C_{K}$ fluorescence, at $277 \mathrm{eV}$, as well as $\mathrm{N}_{\mathrm{K}}$ at $392 \mathrm{eV}$ and $\mathrm{O}_{\mathrm{K}}$ at $525 \mathrm{eV}$, can be particularly informative. These studies provide fundamental understanding that ultimately improves the quality of catalysts, polymers, lubricants, superconductors, organic electronics and biological materials, to name just a few. In the field of nearedge extended X-ray absorption fine structure (NEXAFS), a beam of soft X-rays is incident on the sample of interest (Fig. 1). The number and energy of photo-electrons and fluorescent photons that are emitted from the sample permit sensitive characterization of its elemental structure and chemical bonding. The ability to sweep the incident photon beam through a range of energies, plus the different depth sensitivities of electron emission and photon emission, make NEXAFS studies a most important tool in materials science. Fluorescent photon detection in the few hundred $\mathrm{eV}$ range is challenging because of the small signal amplitude, and additional constraints of UHV compatibility, good solid-angle coverage and long-term stability, present other constraints. We report here on the development of a new sensor for fluorescent photons based on their conversion in low pressure gas, together with electron multiplication using Gas Electron Multipliers (GEMs) [1].

Manuscript received November 20,2009. This work was supported in part by the U.S. Department of Energy under Contract No. DE-AC02-98CH10886.

N.A. Schaknowski is with the Instrumentation Division, Brookhaven National Laboratory, Upton, NY 11973 USA (telephone: 631-344-4261, e-mail: neil@bnl.gov).

G.C. Smith is with the Instrumentation Division, Brookhaven National Laboratory, Upton, NY 11973 USA (telephone: 631-344-4253, e-mail: gsmith@bnl.gov).

B. Yu is with the Instrumentation Division, Brookhaven National Laboratory, Upton, NY 11973 USA (telephone: 631-344-5184, e-mail: yubo@bnl.gov).

D.A. Fischer is with the Materials Science and Engineering Laboratory, National Institute of Standards and Technology, Gaithesburg, MD (telephone: 631-344-5177, e-mail: dfischer@nist.gov).

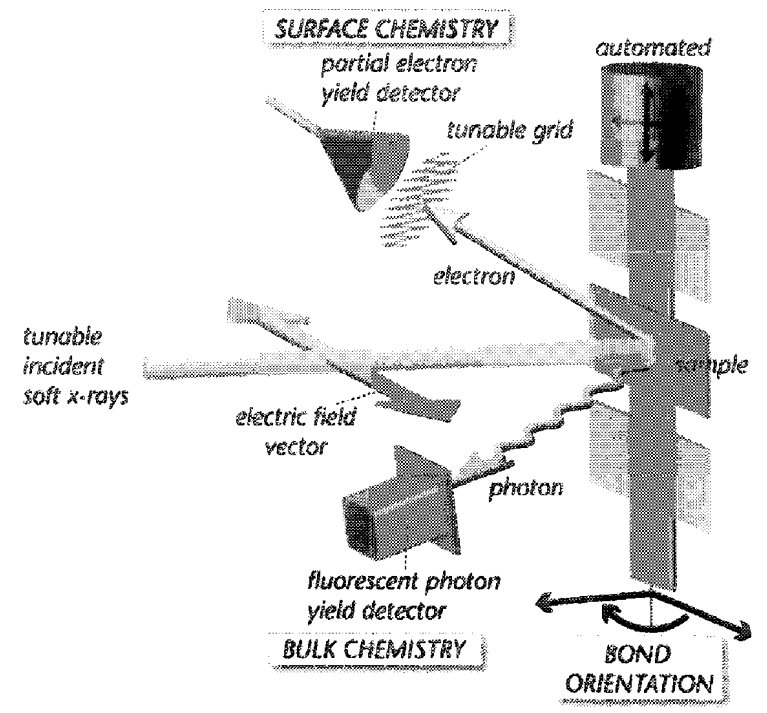

Fig. 1. Diagram illustrating the basics of $x$-ray absorption spectroscopy. An intense, tunable beam of $x$-rays is incident on the sample of interest, and sensors very close-by measure electron and photon emission (photo-electrons and fluorescence photons respectively). Of particular interest in the present development work is a fluorescent photon detector suitable for $\mathrm{C}_{\mathrm{K}}(277 \mathrm{eV})$, $\mathrm{N}_{\mathrm{K}}(392 \mathrm{eV})$ and $\mathrm{O}_{\mathrm{K}}(525 \mathrm{eV})$.

\section{Detector Concept}

The key properties required of the fluorescent photon detector are:

i) UHV compatibility

ii) compact enough to fit into "busy" vacuum chamber

iii) long term stability

iv) energy resolving capability

v) high efficiency

vi) potential for select band-pass operation

vii) large solid angle coverage

Existing sensors that have been used are proportional counters, CsI coated electron multipliers, and semiconductor drift detectors. Electron multlipliers such as Channeltrons and micro-channel plates are available commercially, are relatively easy to use, but have no energy resolving capability and exhibit efficiency that varies rapidly with photon energy. Silicon drift detectors perhaps best satisfy the key requirements, but as yet are not readily available for soft $\mathrm{X}$-ray detection.

Our groups at Brookhaven and NIST have designed and fabricated gas-filled detectors for a number of years, and in fact reported some of the earliest detectors for fluorescence measurements $[2,3]$. For the present studies, we have opted to pursue a design based on electron multiplication using GEMs in a low pressure gas. For photon energies of a few hundred 
$\mathrm{eV}$, it is easy to obtain high stopping power in an argon based gas for $\mathrm{C}_{\mathrm{K}}(277 \mathrm{eV}), \mathrm{N}_{\mathrm{K}}(392 \mathrm{eV})$ and $\mathrm{O}_{\mathrm{K}}(525 \mathrm{eV})$. A key issue is containing the gas, and developing an entrance window that is thin enough to transmit X-rays into the gas, yet does not permit the gas to diffuse into the UHV chamber.

We have been developing GEMs by drilling holes in copper clad insulating material, along the lines pioneered by Amos Breskin for thick GEMs (THGEMS) [4]. Fig. 2 shows a diagram of the detector designed here for fluorescence studies.

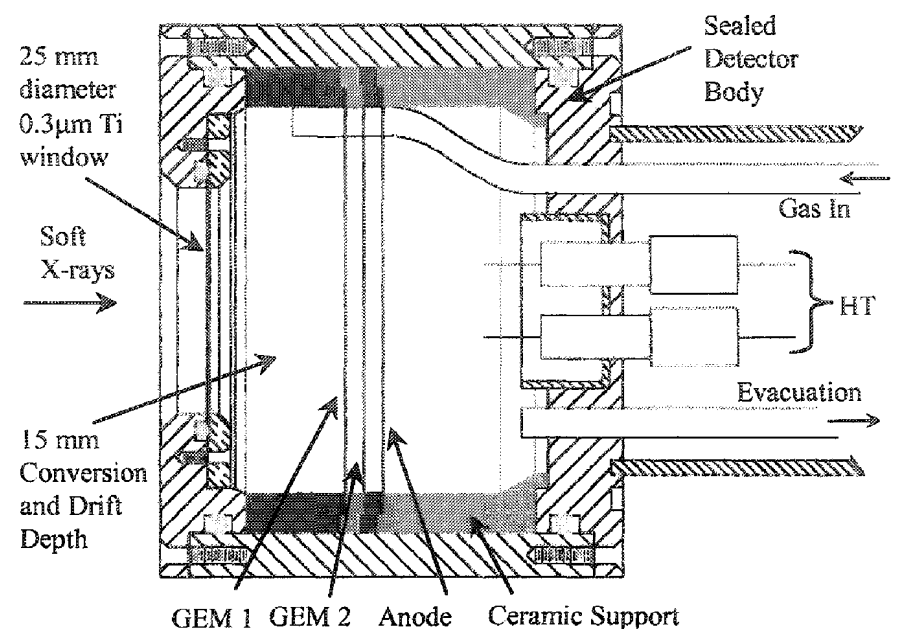

Fig. 2. Diagram of internal arrangement of Gas Electron Multiplier (GEM) electrodes, anode, and sealed detector body. GEMs and anode are spaced $2 \mathrm{~mm}$ apart, each one $200 \mu \mathrm{m}$ thick and comprising $200 \mu \mathrm{m}$ diameter holes on a $450 \mu \mathrm{m}$ pitch.

Photons enter the detector through a $25 \mathrm{~mm}$ diameter window, composed of $300 \mathrm{~nm}$ titanium foil, which is supported on a stainless steel disc with holes that represent a $60 \%$ open area. Behind the window is a $15 \mathrm{~mm}$ conversion and drift region, followed by two GEMs operating in series, and an anode that collects the avalanche charge. In terms of bias, the window is ground, with progressively increasing negative bias applied to GEM1, GEM2 and the anode. Signal from the anode is collected by a charge-sensitive front-end preamplifier, followed by shaping (ORTEC 460 delay line amplifier) and pulse height analysis and counting. Voltage is applied via one of the HT feedthroughs, a resistor chain providing individual bias to each electrode.

\section{A. GEMs}

Our goal was a fabrication process which could be undertaken in-house. The most commonly used GEMs, copper coated kapton, require a liquid etching technique that we have not yet attempted to develop. We have been intrigued by the THGEM technique [3], particularly as we have high speed drilling capability for regular printed circuit boards. Thus, we chose to investigate GEMs fabricated from Rogers 4000 circuit board material [5], but keeping the thickness to a minimum, thereby minimizing operating voltage. The smallest diameter hole that can be reliably drilled many times over is $200 \mu \mathrm{m}$, and in order to maintain a hole diameter to depth ratio of around unity (the typical value for many GEMs), the starting material chosen was $200 \mu \mathrm{m}$ thick. Fig. 3 shows a photograph of the manufactured GEMs (on the right), and macor spacers and supporting rings on the left. Fig. 4 shows a close-up of the holes drilled in the GEMs, highlighting the uniformity, well-defined cylindrical nature of the holes, and etching of copper on the underside.

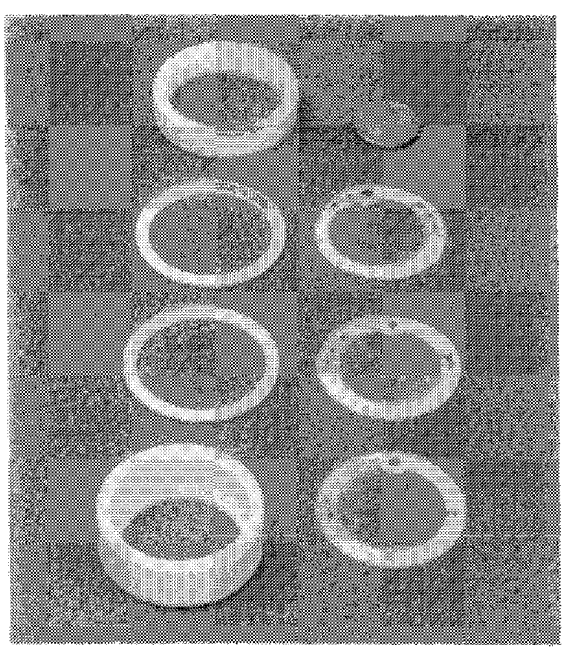

Fig. 3. Photograph of ceramic spacers and supports rings (left), and Gas Electron Multipliers plus anode (right). A US quarter, top right, shows scale.
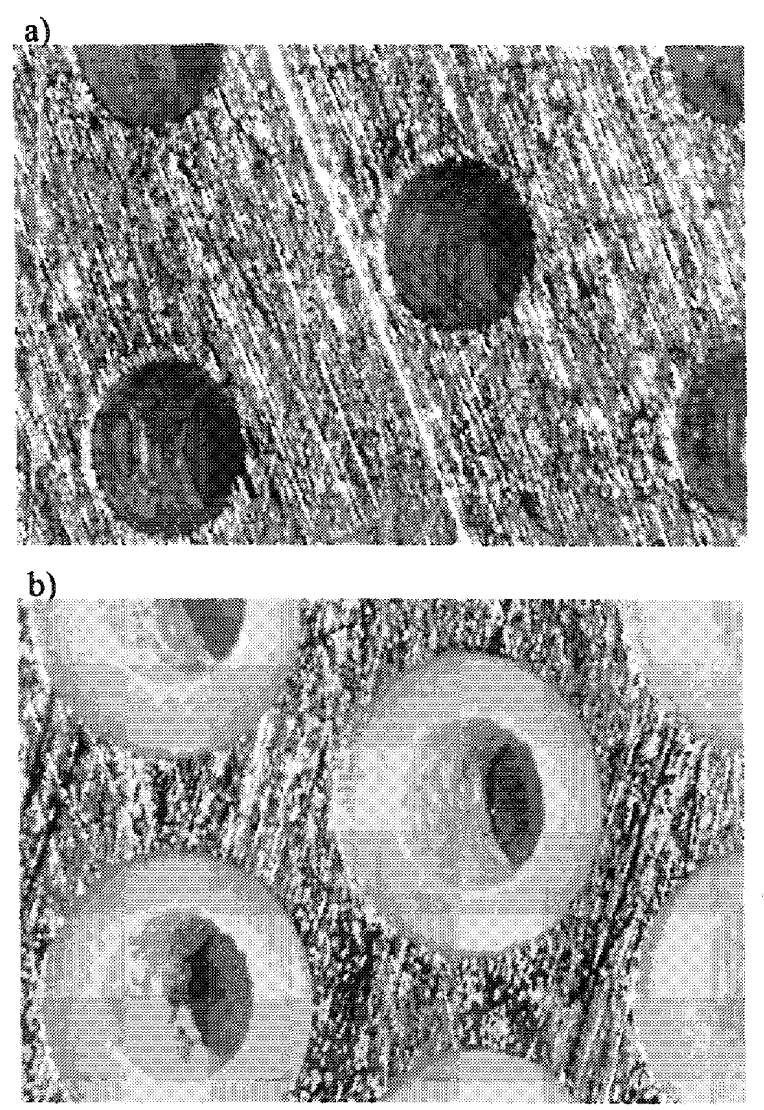

Fig. 4. Close-up photographs of holes in GEM electrodes. a) shows top of GEM electrodes, b) shows bottom of GEM electrodes, where a $50 \mu \mathrm{m}$ wide annulus has been etched around all holes to increase the surface leakage path between top and bottom electrodes. GEM electrodes are $200 \mu \mathrm{m}$ thick and comprise $200 \mu \mathrm{m}$ diameter holes on a $450 \mu \mathrm{m}$ pitch. 


\section{B. Titanium Window}

The most common materials for thin-windowed gas detectors are plastics, such as mylar and polypropylene $[2,3]$. However, these materials generate a big change in photon transmission at the carbon edge, making analysis and normalization of NEXAFS data problematic. As an alternative, titanium has been used in this application. The window consists of three layers of $100 \mathrm{rm}$ titanium foll, resting on a stainless steel support disc. The individual titanium sheets contain a small number of minute pin-holes, but three layers together essentially represent a total barrier to gas diffusion. Fig. 5(a) shows the assembled window frame, and Fig. $5($ b) a close-up of the unstressed titanium.
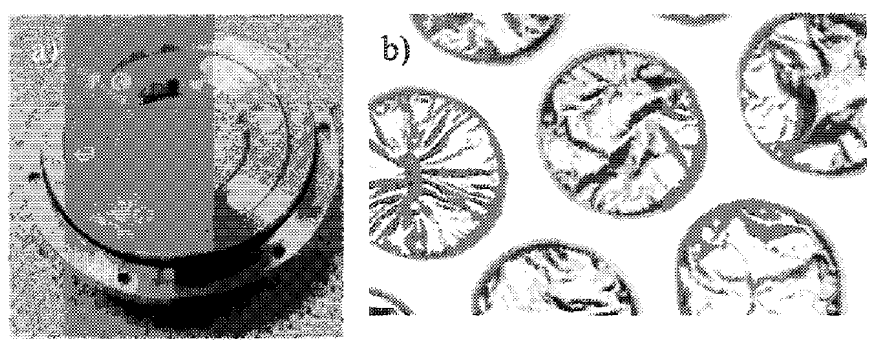

Fig. 5. Photographs of titanium window. a) shows detector side, with titanium foil facing the reader - sensitive diameter is $25 \mathrm{~mm}$. b) shows vacuum side with titanium non-stressed by pressure differential - support is provided by a stainless steel disc with holes of diameter $1.15 \mathrm{~mm}$, on a spacing of $1.41 \mathrm{~mm}$, representing an open area of approximately $60 \%$.

The fully assembled detector forms a compact device that is UHV compatible at the working gas pressure. Fig. 6(a) shows the full internal assembly and Fig. 6(b) the complete assembly.
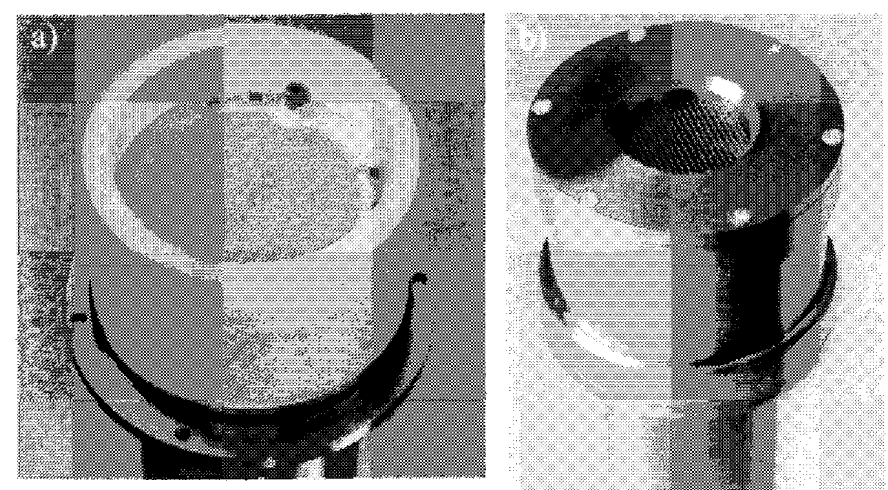

Fig. 6. Photographs of detector assembly. a) shows fully assembled GEMs and ceramic spacers (only the top of GEM1 is visible). The blue-colored chips are part of the HT resistor chain, which is grounded via a final connection to the gas pipe. b) shows complete assembly with body and window in place.

\section{Operaitonal characteristics}

Several competing elements contribute to the optimum operating pressure. Obviously, there needs to be adequate stopping power (pressure) for the X-ray energy in question, but reducing the probability of window rupture and minimizing diffusion of gas into the vacuum chamber require a pressure as low as possible. However, it becomes electrically very difficult to operate this electrode configuration below 50 torr, and an efficient gas mixture for $\mathrm{C}_{\mathrm{K}} \mathrm{X}$-rays in $\sim 15 \mathrm{~mm}$ was chosen $\left(30 \% \mathrm{Ar} / 70 \% \mathrm{CH}_{4}\right)$. Fig. 7 shows the efficiency of $15 \mathrm{~mm}$ of 50 torr of this gas, and around the $C_{K}$ edge, the stopping power is nearly $100 \%$. However, the full efficiency of the detector should include the window transmission: Fig. 7 also shows the titanium transmission, taking into account the $60 \%$ open area of the stainless steel support. The product of these two curves (solid purple curve) represents the complete detector efficiency. As a reminder of edge energies and fluorescent line energies, these are shown in Table I. Fig. 8 shows the calculated efficiency for $15 \mathrm{~mm}$ vs pressure of $30 \% \mathrm{Ar} / 70 \% \mathrm{CH}_{4}$. All efficiency/transmission calculations have been performed using the Lawrence Berkeley Laboratory web-resource [6].

TABLE I. ELECTRON BINDNG ENERGES/LINE ENERGIES OF INTEREST

\begin{tabular}{lccccc} 
& $\mathrm{C}_{\mathrm{K}}$ & $\mathrm{N}_{\mathrm{K}}$ & $\mathrm{O}_{\mathrm{K}}$ & $\mathrm{Ar}_{\mathrm{L}}$ & $\mathrm{T}_{\mathrm{L}}$ \\
\hline Electron binding energy $(\mathrm{eV})$ & 284 & 410 & 543 & 248 & 454 \\
Characteristic line $(\mathrm{eV})$ & 277 & 392 & 525 & &
\end{tabular}

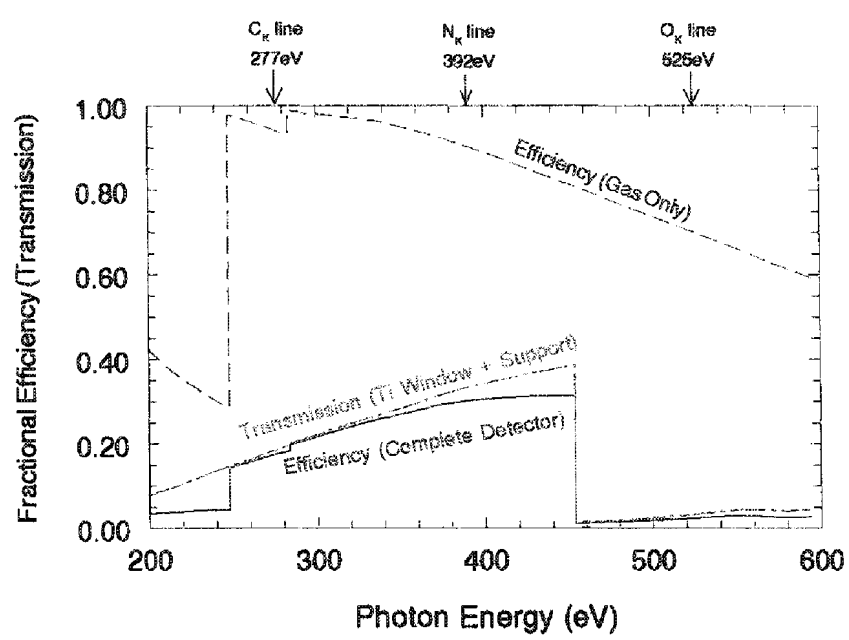

Fig. 7. Calculated efficiency of detector, as a function of photon energy. Red (even dashed) curve represents fractional absorption in $15 \mathrm{~mm}$ gas depth only, blue (uneven dashed) curve represents transmission of $0.3 \mu \mathrm{m}$ titanium window plus its support, and purple (solid) curve is the product of the two.

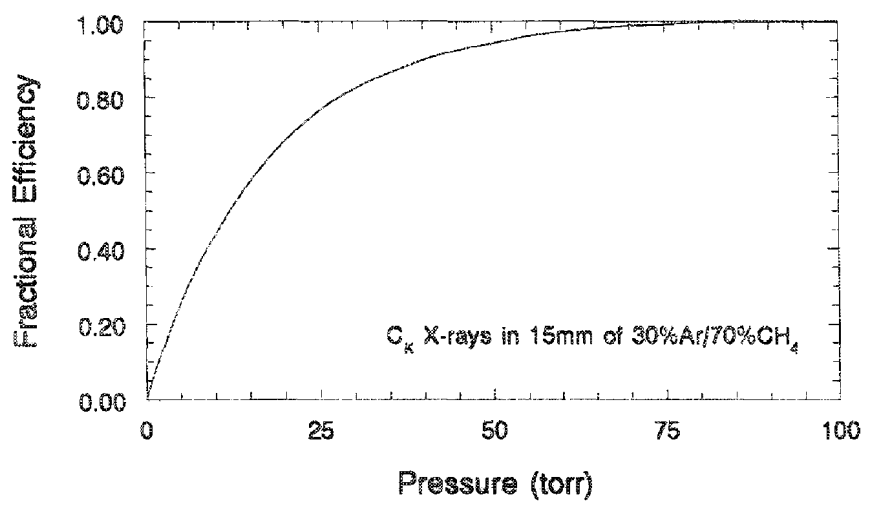

Fig. 8. Calculated efficiency of detector gas only, as a function of pressure, for $\mathrm{C}_{\mathrm{K}} \mathrm{X}$-rays in 50 torr of $30 \% \mathrm{Ar} / 70 \% \mathrm{CH}_{4}$. 
Clearly, at 50 tor and above the efficiency is essentially unity, but below 30 or 40 tor the efficiency begins to fall rapidly - thus 50 tont is a good balance between efficiency and total stress on the window.

At low pressures of a few tens of torr, if the gas gain exceeds a certain threshold, some form of ion-induced feedback occurs that creates operational instability. Fig. 9 illustrates the mode signals waveforms as this instability develops.
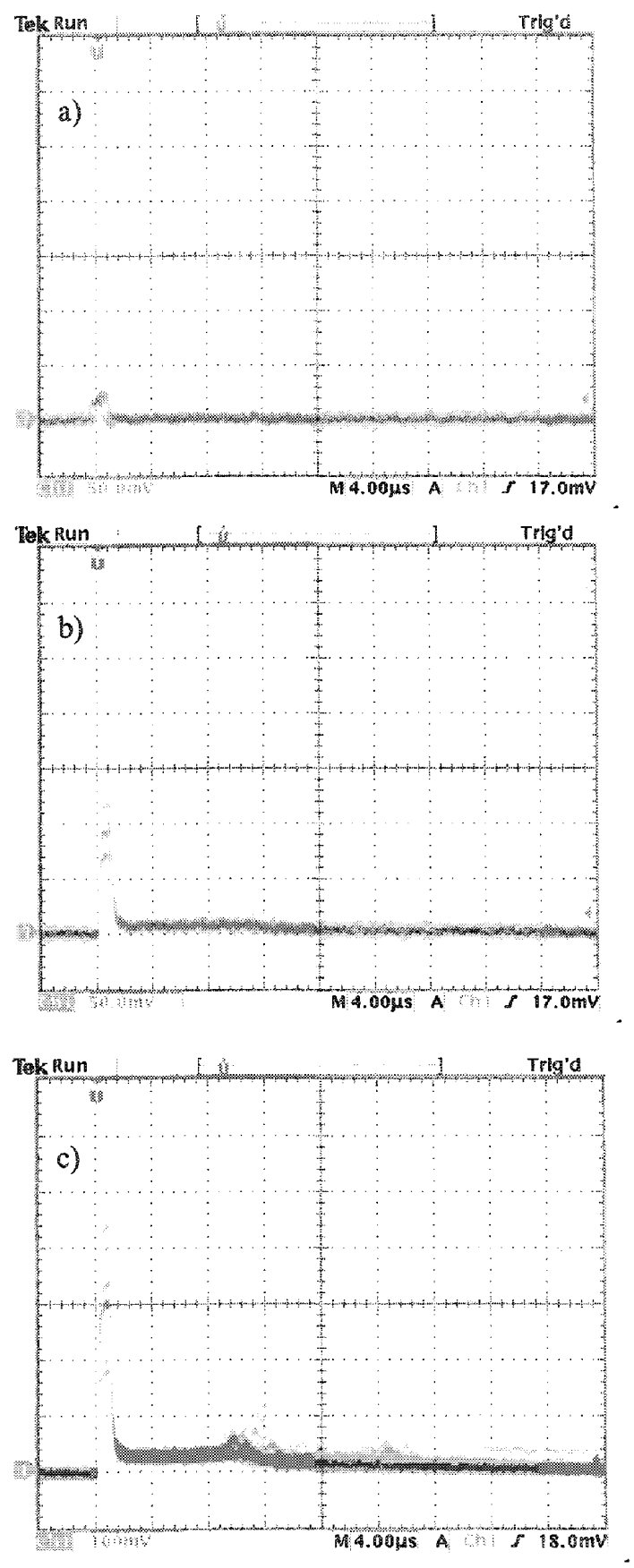

Fig. 9. Wavefoms from anode as a function of gas gain, with 50 tor of $30 \% \mathrm{Ar} / 70 \% \mathrm{CH}_{4}$ :

a) Effective Gain $=1500$ (vertical scale $50 \mathrm{mV} /$ div)

b) Effective Gain $=6000$ (vertical scale $50 \mathrm{mV} /$ div)

c) Effective Gain $=18000$ (note change in vertical scale to $100 \mathrm{mV} /$ div)
In Fig 9(a), the effective GEM gain is $\sim 1500$, creating about 15000 electrons on average, marginally clear of the electronic noise of around $1000 \mathrm{e}$ FWHM. At a gain of 6000 , the signal safely exceeds the baseline noise, and generating valid counts above a preset theshold is straightforward. As the gain is increased further, however, small signals begin to appear about $10 \mu \mathrm{s}$ after the main signal, a time that is consistent with the drift of positive ions from the anode to GEM2. It also appears from the waveform in Fig 9 (c) that some level of parallel plate multiplication is occurring in this gap, as seen by the small positive offset during that 10 us. This phenomenon is not mderstood, and is receiving furher examination. It should be noted that with operating pressure much below 50 torr, it is not possible to achieve any measurable signals before instability occurs. Conversely, above 75 tor, the behavior is stable even for gains of 50,000 .

Under the conditions of fig $9(b)$, the detector is stable and linear, as shown by the analysis of $\mathrm{C}_{\mathrm{K}}$ and ${ }^{55} \mathrm{Fe}$ spectra in Fig. 10. For $\mathrm{C}_{\mathrm{K}} \mathrm{X}$-rays, resolution is reasonable, around $80 \%$ FWHM, and shows little dependence on shaping times over the range 0.25 to $2.0 \mu$ s.

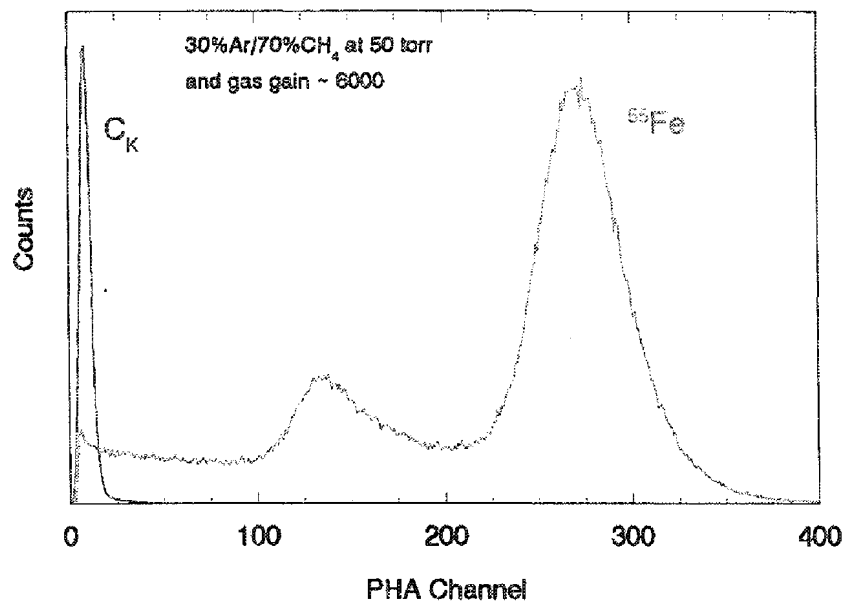

Fig. 10. Pulse height spectra for $C_{K}$ and ${ }^{55} \mathrm{Fe}$ at a gas gain of about 6000 , showing good linearity and energy resolution.

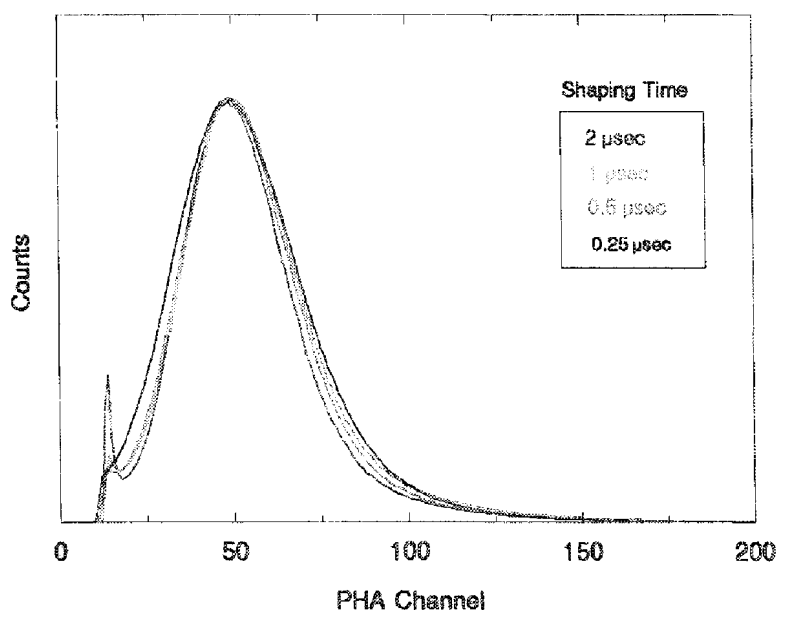

Fig. 11 Pulse height spectra for $C_{K}$ at a gas gain of 6000 , for various shaping times (electronic gain normalized to remove pulse height reduction as time constant decreased). Energy resolution is about $80 \%$ FWHM. 


\section{NEXAFS MEASUREMENTS}

The soft $X$-ray detector is now installed on beam-line $X 7 \mathrm{~A}$ of the NSLS, an X-ray absorption spectroscopy beam-line managed by NIST. Initial testing has been carried out with a set-up similar to that in Fig. 1, with the fluorescent photon yield detector represented by the soft $\mathrm{X}$-ray detector described in this work. Measurements have been made on a variety of NIST standard samples, and Fig. 12 illustrates two such

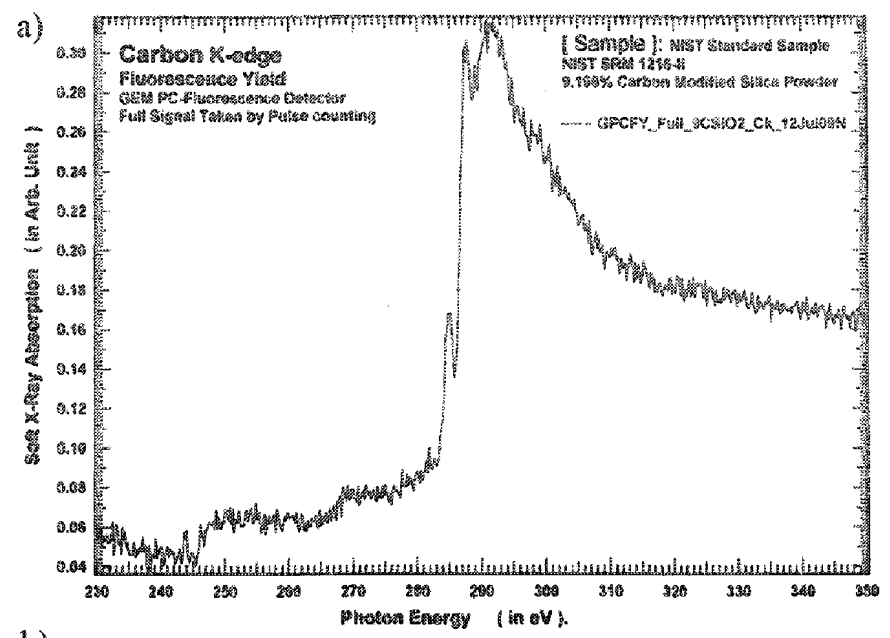

b)

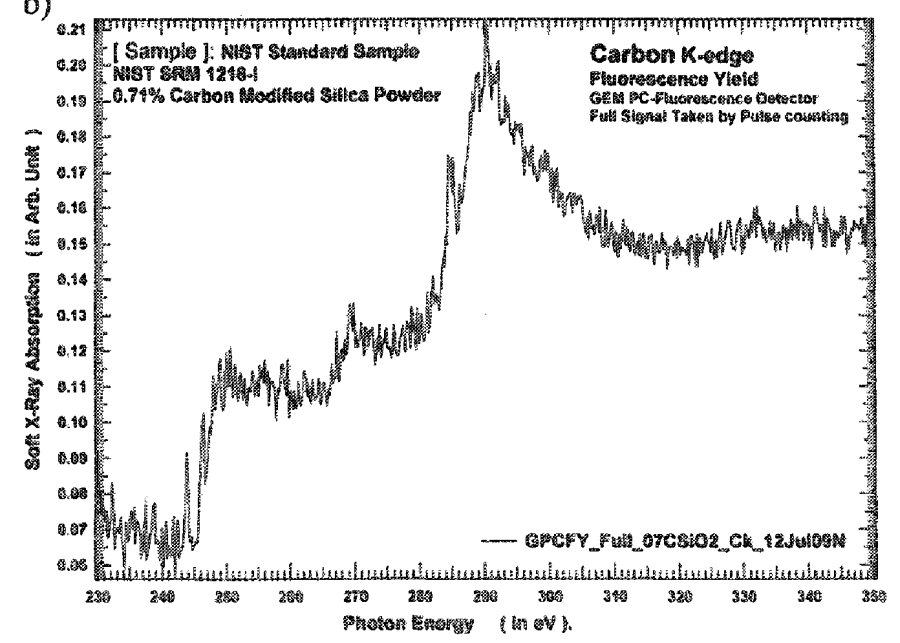

evaluations.

Fig. 12. Carbon NEXAFS results from NIST standard samples.

The samples consist of carbon modified silicon powder. In these measurements, the incident primary beam from the synchrotron source was swept in energy from $230 \mathrm{eV}$ to $350 \mathrm{eV}$, and the count rate registered from the soft $\mathrm{X}$-ray detector was continuously recorded. Fig. 12(a) and 12(b) shows counts as function of beam energy for a sample containing $9 \%$ and $0.7 \%$ by weight of carbon, respectively. As the beam energy sweeps through the carbon $\mathrm{K}$-edge at $284 \mathrm{eV}$, there is an obvious increase in counts, due to generation of $\mathrm{C}_{\mathrm{K}}$ fuorescence. Even when the primary beam energy is below the carbon edge, the defector count rate is non-zero, due mainly to detection of scattered photons from the sample. As expected, the sample containing less carbon produces a smaller fluorescence flux, but in both examples the peak structure in the rising edge at
$284 \mathrm{eV}$ is an intrinsic feature of $\mathrm{C} \cdot \mathrm{C}$ bonding in the sample. An increase in counting rate also occurs at the argon L-edge $(248 \mathrm{eV})$, due to the increase in the argon absorption crosssection above $284 \mathrm{eV}$, and it is interesting to note that the peak structures that occur at the rising edge at $284 \mathrm{eV}$ are also due to fine structure in the argon electron binding energies.

We have determined from several sets of experiments that the detector represents a well-sealed and clean intenal enviromment for the gas mixture. The diagram in Fig. 2 shows that, in the initial design, the gas system was in flow mode. We are now able to cvacuate the detector, back-fill with 50 tor of $30 \% \mathrm{Ar} / 70 \% \mathrm{CH}_{4}$ from a single gas-line, valve off that line, and run for over a week without any noticeable change in gas gain or energy resolution.

\section{ACKNOWLEDGMENT}

We are grateful for the help of Gene von Achen, Ron Angona, Howard Hansen and Ron Ryan (BNL) in designing aud fabricating developmental versions of Rogers 4000 GEMs. We thank Zugen Fu and Chemo Jaye (NIST) for assistance with the NEXAFS measurements. We appreciate the support and encouragement of Veljko Radeka (BNL) during this project.

\section{REFERENCES}

[1] F. Sauli, "GEM: A New Concept for electron Amplification in Gas Detectors", Nucl. Instrum. \& Meth. A386 (1997) 531-534.

[2] D.A. Fischer, J. Colbert and J.L. Gland, "Ultrasoft (C, N, O) X-ray fluorescence detection: Proportional counters, focusing multilayer mirrors, and scattered light systematics", Rev. Sci. Instrum. 60 (1989) 1596-1602.

[3] G.C. Smith, A. Krol, and Y.H. Kao, "A Low-pressure, Parallel Plate Avalanche Chamber for Soft X-ray Fluorescence", Nucl. Insirum. \& Meth. A291 (1990) 135-139.

[4] A. Breskin, R. Alon, M. Cortesi, R. Chechik, J. Miyamoto, V. Dangendorf, J.M. Maia, and J.M.F. Dos Santos, "A Concise Review of THGEM Detectors", Nucl. instrum. \& Meth. A598 (2009) 107-111.

[5] Rogers Corporation: Rogers 4000 is low out-gassing, low dieletric constant, high frequency circuit material.

[6] http:/henke.lbl.gov/optical_constants/ 